\section{Why Medical Informatics (still) Needs Cognitive and Social Sciences}

\author{
G. Declerck, X. Aimé, Section Editors for the IMIA Yearbook Section on Human Factors and \\ Organizational Issues \\ French National Institute of Medical Research and Public Health, INSERM, UMRS 872 EQ 20, \\ Knowledge Engineering for HealthCare
}

\section{Introduction}

This year's selection of papers addressing Human Factors and Organizational Issues (HFOI) focuses on the use of paradigms, methodologies, models, and results from cognitive and social sciences in medical informatics, i.e. on the question of interdisciplinarity.

Medical informatics is not originally concerned with how cognition works, or even with how technology impacts cognition, but with building efficient tools, facilitating the work of health practitioners (HPs), making it more efficient, and improving the quality of care. To achieve this goal, researchers try to design systems facilitating HPs' access to data and knowledge, supporting their diagnostic or therapeutic decisions, and increasing their understanding of healthcare situations. But as the HFOI section claims now for several years, building tools able to augment efficiently cognition (e.g. decision-making in complex situations) cannot succeed without understanding how cognition works in situ, and how the appropriation of tools impacts the way cognition works. As Berg [1-3] explains, when a developer creates a new tool, she not only provides new - hopefully - useful functionalities, but potentially changes work practices of all involved users. "Technology deployments, such as the implementation of a patient care information system, are [...] a process of mutual transformation between the organization and the technology rather than merely [...] a matter of bringing an automated tool into a working environment." [4] Studying end user cognition and sociotechnical issues is consequently an essential ingredient for achieving and implementing usable tools [5-7]. In order to succeed at its mission, medical informatics has no other option than to work closely with cognitive and social sciences.

Several cross-disciplinary activities have already been undertaken in medical informatics and the field of Human Factors is growing stronger each year. Medical informatics researchers are regularly using observational methods from sociology such as the ethnomethodological framework [9] or cognitive psychology models for the decision making process. Initiatives have also been made to reinforce the role of HFOI in medical informatics such as the IMIA working group on human factors engineering in health informatics lead by Peter L. Elkin and Marie-Catherine Beuscart-Zephir, whose objectives are, among others, "to promote methods and techniques devoted to the study of human factors in the field of Biomedical Informatics" and "to coordinate studies and actions in this domain and to develop standardization initiatives for usability studies and user-centered design in the healthcare domain" [10].

We think, however, that further efforts in Human Factors research still need to be made. Health Information Technology (HIT) adoption rates remain low [6] and complete adoption failures are reported regularly [11-13]. In addition, the positive impact of the vast majority of implemented HIT on the quality and safety of healthcare has still to be demonstrated [14]. For several researchers, this situation is explained in part (or more) by the persistent technocentrism in HIT engineering and the seemingly unbridgeable "gulf between technologic artifacts and end users" [7]. 
Although it has been repeatedly demonstrated that a lot of extra-technological factors influence HIT adoption, among which - to mention only a few - established work practices, social structures, communication infrastructures, relationships between stakeholders, cultural factors, and cognitive factors (e.g. cognitive load issues or cognitive consequences to tasks interruption) [15-20], most medical informatics projects are mainly or exclusively focused on technological issues (e.g. on the famous interoperability problem), dealing with aforementioned factors only as peripheral elements. When looking at medical informatics projects that have been funded by the European Union's Seventh Framework Programme for Research (FP7) in past years, one can easily see that very few include teams with expertise in the field of cognitive or social sciences [21]. At best the engineering teams in charge of building the prototype have some notions of GUI ergonomics. This technocentrism is not only counterproductive for medical informatics researchers (whose tools are not used), it is also a dramatic waste of resources: As it is often claimed, this funding could be used for beds, employees, facilities, services...

The 2013 HFOI papers selection has been made with the intention to change this landscape and push medical informatics one step closer towards interdisciplinarity. We hope that this selection will contribute to make researchers aware of the necessity of building medical informatics projects with a strong interdisciplinary character, involving researchers from cognitive sciences and sociology, and trying to take into account how end user cognition works, not inside the brain but in situ, embedded in a body relying on tools, interacting with other stakeholders, and dealing with physical as well as ethical and socio-normative constraints.

\section{Best Papers Selection}

The six selected papers illustrate the central role cognitive and social sciences can play in medical informatics research.

Studying cognition does not necessarily mean studying what is happening in the brain

Table 1 Best papers selection of articles for the IMIA Yearbook of Medical Informatics 2013 in the section 'Human Factors and Organizational Issues.' The articles are listed in alphabetical order of the first author's surname.

Section

Human Factors and Orginazational Issues

- Djulbegovic B, Hozo I, Beckstead J, Tsalatsanis A, Pauker SG. Dual processing model of medical decision-making. BMC Med Inform Decis Mak 2012 Sep 3;12:94.

- Li SY, Magrabi F, Coiera E. A systematic review of the psychological literature on interruption and its patient safety implications. J Am Med Inform Assoc 2012 Jan-Feb;19(1):6-12.

- Park SY, Lee SY, Chen Y. The effects of EMR deployment on doctors' work practices: a qualitative study in the emergency department of a teaching hospital. Int J Med Inform 2012 Mar;81(3):204-17.

- Pelayo S, Anceaux F, Rogalski J, Elkin P, Beuscart-Zephir MC. A comparison of the impact of CPOE implementation and organizational determinants on doctor-nurse communications and cooperation. Int J Med Inform 2012 Sep 20.

- Schraagen JM, Verhoeven F. Methods for studying medical device technology and practitioner cognition: The case of user-interface issues with infusion pumps. J Biomed Inform 2013 Feb;46(1):181-95.

- Tariq A, Georgiou A, Westbrook J. Medication errors in residential aged care facilities: A distributed cognition analysis of the information exchange process. Int J Med Inform 2013 May;82(5):299-312.

of the subject when he is performing a reasoning task. This internalist and brain-centric vision of cognition is undoubtedly still vivid (and prevailing) in cognitive sciences, but several alternative paradigms are now available, among them $(i)$ the distributed cognition framework [22-26], which moves the unit of analysis from the brain to the overall system possibly comprising several individuals (with their brains) exchanging information through several communication channels and relying on artifacts and environmental structures to achieve tasks; and (ii) the situated, externalist and enactivist approaches to cognition [27-31], which insist on the role played by the body, tools, and interactions with the environment in cognitive performances. Several medical informatics researchers have used these approaches, especially the distributed cognition paradigm, to model and understand clinical care situations, where the distributed character of cognitive tasks is particularly pronounced. This is the case in the two first selected papers.

The first paper [32] makes use of the so-called Cognitive Systems Engineering approach to study user-interface issues with infusion pumps. The core principle of this approach, based on Donald A. Norman user-centered design [33], is to study artifacts not as objects, but as systems that shape cognition and collaboration. Using an impact flow diagram built on the basis of a review of literature on infusion pumps' user interface issues, the authors analyze a situation (described in [34]) of infusion pump use failure (morphine concentration programming error). Their analysis demonstrates that the classical "human error" explanation of such incident (narrow focus on individual cognition) is not appropriate because it hides the role factors like the organizational structures or distributed representations play in such interface use failures. The authors propose to use instead a systemic approach considering the system as a whole, i.e. thinking "of people and technology not as independent components, but rather as a single ensemble where breakdowns in coordination may occur". One of the most interesting contributions of the paper is the "keyhole effect" concept, which refers to the access to piecemeal information in situations where different HPs take care of the same patient for brief periods of time: The HPs "merely get a glimpse of a more dynamic and continuous process to which the patient [has] more privileged access". The authors show that this effect, which is the consequence of organizational policies and regulations, is partly responsible for the infusion pump incident analysed. 
The second paper [35] makes use of a distributed cognition framework to analyze information exchanges in residential aged care facilities (RACFs), which lead to medication errors. In line with [32], the authors attribute medication errors not to individual care providers, but to failures occurring in a more general distributed cognitive system, including physically distributed participants mediated by cognitive artifacts, in this case to gaps in information exchange processes. Based on a five months ethnographic study including several qualitative methods (minimally intrusive direct observations, semi-structured interviews, and artefact analysis), four interrelated forms of cognitive distribution (social, structural, spatial, and temporal) implicated in the medication error process were identified. Limitations in the design of medication charts, the key cognitive artefact for coordinating the medication process, were also identified as causing information exchange gaps. In the end, the authors demonstrate that "the fragmented nature of existing health sectors and their limited capacity to function as part of a large cognitive system" is the first cause of medication errors, and that coordination mechanisms are consequently needed to reduce the existing variability of the process.

The third paper [36] also addresses organizational issues and the impact of HIT on information exchange and coordination processes. Using in situ observations and interviews, the authors studied the impact of Computerized Physician Order Entry (CPOE) implementation on doctor-nurse cooperation and communication processes in different workplace organizations (common round, briefing, opportunistic exchanges). The main result of the study, which contradicts a widespread idea, is that the CPOE system had no significant impact on the cooperative activities and communication processes, which are influenced only by the workplace organizations. This does not mean that HIT may not have indirect consequences on those processes. As stated by the authors, "it is very likely that the introduction of a CPOE may provoke a major change in terms of the organisation, for instance progressively shifting from a com- mon round organisation to an opportunistic one", and "this can have a negative effect on nurse-doctor communications". But the effect will be mediated by the transformation of the workplace organizations. This result is important because it suggests that to minimize the possible negative effects of HIT on communication between actors, it is not on the technology that one must act, but on the workplace organizations, trying to maintain organization structure that are not deleterious for information exchanges and coordination.

The two next papers illustrate how models and empirical data from cognitive psychology can be used in medical informatics.

The fourth paper [37] is impressive by its strong theoretical background and the quantity of empirical results covered. The authors make use of Mukherjee's Dual System Model [38] (based on Daniel Kahneman's dual processing theory of cognition [39]) to model the cognitive process of deciding whether treatment should be administered in situations where diagnosis is not fully decided (the patient may or may not have a disease). A threshold probabilistic model taking into account - among other factors - risk assessment, affect-based judgments, recent experience of the physician, time-pressure, data available, memory limitations, and anticipation of post-choice regrets is developed to describe how system I and system II interact to make a treatment decision. A detailed application to classical medical situations (treatments for pulmonary embolism and acute leukemia) demonstrates that the model can be used to better understand decision-making at the bedside and illustrates how it can explain the widespread variation in treatments observed in clinical practice. One important contribution of the paper is the formalization of the role emotional determinants play in medical decision making. The model especially explains how efforts to minimize anticipated regret can make physicians' decisions deviate from rational choice (e.g. as formalized by expected utility theory). The only potential weakness of the paper is that issues related to HIT design are not discussed. In principle the utility of this model for Decision Support Systems design is quite obvious, but a concrete implemen- tation might encounter challenges. Further work is needed to address this issue.

The fifth paper [40] offers a systematic review of experimental studies on interruption in psychology and human-computer interaction to understand the effects of interruption in healthcare. Based on a solid theoretical background, the review shows that interruption has a negative effect on task completion time, resumption lag, work strategy, decision-making process, and errors, and that interruption effects depend on the type of clinical task (procedural, problem-solving, or decision-making). Twelve variables that influence interruption effects are identified. Among the most important are working memory load, interruption position, similarity, modality, handling strategies, and practice effect. For instance, interruption at high working memory load is usually associated with decreased primary task performance. An interruption that demands the same cognitive mechanism as the primary task is also more likely to disrupt task performance, because of interference-inhibiting effects. Several recommendations to minimize the disruptive effects of interruption in clinical settings and to design work processes and information systems that are resilient to interruption effects are made. The paper demonstrates the interest of taking into account psychological models and empirical results in the design of HIT.

The last paper [41] is particularly important because it illustrates (once more) the kind of unexpected side effects HIT can have on cognitive and work processes. Using a six-month qualitative study (in situ field observations and semi-structured interviews), the authors studied how an EMR (Electronic Medical Record) system affects medical work practices (especially documentation practices) by observing the rollout of an EMR in an Emergency Department during the paper-to-electronic transition period. Several important drawbacks of the EMR are highlighted: Increasing the cognitive burden of doctors instead of alleviating it, aggravating HPs' multi-tasking due to flexibility in the system use, which allows for more interruptions, and causing HPs' work to become largely stationary in the charting room, thus reducing the time 
spent with patients and the interaction with nurses. This last point confirms the results of other studies demonstrating that HIT can induce an important loss of psychosocial information [4, 42].

This paper also shows an original approach in the field of HFOI because it deals with issues addressed by an unfortunately little known field of cognitive sciences: cognitive technologies studies. This field - based on the original work of the anthropologist Jack Goody [43] - analyzes the cognitive properties of symbolic technologies, i.e. technologies to store and communicate information. Typically, writing enables a (quasi-)permanent encoding of the message, without alteration of its content, unlike the oral communication channel [43]. The ubiquity of the electronic document, i.e. the possibility to be accessed and updated from several places at the same moment, is one of those remarkable cognitive properties. This contrasts with the "uniquity" of the paper document, which exists as one single instance at one single place at a time. But ubiquity is not necessarily desirable when dealing with patient record. Typically the authors observed that because of the uniqueness and limited spatial access to paper records, "doctors always tried to finish their documentation and make paper charts available for use as quickly as they could". In contrast, the flexibility of electronic charting, which enables to pause, resume, and save, leads to increased multi-tasking in the documentation task, and possibly to increased cognitive load. The uniqueness of the paper record also has an indirect positive effect on communication. Because "they had to go to the nursing stations to pick up the paper charts $[\ldots]$, the doctors naturally interacted with nurses and spoke about the progress of their patients whenever the charts were picked up". Finally, the paper document is also much more portable than computer systems, which do "not support the multiple patient care process and the mobile nature of ED work". Doctors thus "developed a habit of using personal hand-written notes as memory aids to carry bedside information back to the charting room for later documentation, jotting down information during patient interviews."

\section{Paper Selection Method}

Seventeen medical informatics journals (Journal of Biomedical Informatics, Methods of Information in Medicine, etc.), but also Medicine (Drug safety) and Ergonomy (Human factors) were selected (partly based on [44]). Using PubMed, a total of 3,024 papers were evaluated by the two HFOI section editors in the pre-selection phase, on the basis of their title, keywords, and abstract. 73 papers were preselected. The list was further refined to 15 papers, which were carefully evaluated by six referees using the standard evaluation grid of the Yearbook: Attribution of a score from 0 to 100 using 5 criteria: (i) Significance (Topic's importance and Scientific and/or practical impact); (ii) Quality of scientific and/or technical content; (iii) Originality and innovativeness; (iv) Coverage of related literature; $(v)$ Organisation and clarity of presentation. The 6 best rated papers were selected after discussion with the Yearbook editorial committee.

Several inclusion/exclusion criteria were applied during the pre-selection phase:

In general, were excluded: (a) papers focused on the evaluation of a precise system, without general considerations on adoption issues (e.g. recommendations); (b) studies simply measuring user satisfaction; (c) studies only focused on the ergonomics or usability of systems or only dealing with human-computer interactions issues in a narrow sense; (d) studies relying on questionnaires without additional observational methods; (e) review papers.

In general, were included: (a) Studies making use of paradigms, methodologies, models, and empirical results from cognitive or social sciences; (b) studies explicitly involved in the HFOI approach in medical informatics, i.e. recognizing the limitations of the standard engineering approach, only focused on technology; (c) studies discussing theoretical or methodological issues related to the HFOI approach in medical informatics; (d) in situ field observations studies rather than qualitative studies using questionnaires or semi-structured interviews.

\section{References}

1. Berg M, Toussaint T. The mantra of modeling and the forgotten powers of paper: a sociotechnical view on the development of process-oriented ICT in health care. Int J Med Inform 2003;69:223-34.

2. Berg M. Patient care information systems and health care work: a sociotechnical approach. Int J Med Inform 1999;55(2):87-101.

3. Berg M. Implementing information systems in health care organizations: myths and challenges. Int J Med Inform 2001;64(2):143-56.

4. Park SY, Pine K, Chen Y. Local-universality: designing EMR to support localized informal documentation practices. Proceedings of the 2013 conference on Computer supported cooperative work. ACM; 2013. p. 55-66.

5. Horsky J, Zhang J, Patel VL. To err is not entirely human: complex technology and user cognition. J Biomed Inform 2005;38(4):264-6.

6. Karsh BT, Weinger MB, Abbott PA, Wears RL. Health information technology: fallacies and sober realities. JAm Med InformAssoc 2010;17(6):617-23.

7. Lau F. Extending the Infoway Benefits Evaluation framework for health information systems. Stud Health Technol Inform 2009;143:406-13.

8. Patel VL, Kaufman DR. Medical informatics and the science of cognition. J Am Med Inform Assoc 1998;5(6)493-502.

9. Garfinkel H. Studies in ethnomethodology. NJ: Englewood Cliffs; 1967.

10. International Medical Informatics Association (IMIA) website. Presentation of the Human Factors Engineering for Healthcare Informatics Working Group. http://www.imia-medinfo.org/ new2/node/142

11. Heeks R. Information systems and developing countries: Failure, success, and local improvisations. The Information Society 2002;18(2):101-12.

12. Heeks R. Health information systems: Failure, success and improvisation. Int J Med Inform 2006;75(2):125-37.

13. Wears RL, Cook RI, Perry SJ. Automation, interaction, complexity, and failure: A case study. Reliability Engineering \& System Safety 2006;91(12):1494-501.

14. Black AD, Car J, Pagliari C, Anandan C, Cresswell $\mathrm{K}$, et al. The impact of eHealth on the quality and safety of health care: a systematic overview. PLoS Med 2011;8(1):e1000387.

15. Kuziemsky C, Jewers H, Appleby B, Foshay N, Maccaull W, Miller K, et al. Information technology and hospice palliative care: social, cultural, ethical and technical implications in a rural setting. Inform Health Soc Care 2011;37(1):37-50.

16. Campbell EM, Sittig DF, Ash JS, Guappone KP, Dykstra RH. Types of unintended consequences related to computerized provider order entry. J Am Med Inform Assoc 2006;13(5):547-56.

17. Ash JS, Berg M, Coiera E. Some unintended consequences of information technology in health care: the nature of patient care information system-related errors. J Am Med Inform Assoc 2004;11(2):104-12.

18. Whitten P, Holtz B, Meyer E, Nazione, S. Telehospice: reasons for slow adoption in home hospice 
care. J Telemed Telecare 2009;15(4):187-90.

19. Harrison MI, Koppel R, Bar-Lev S. Unintended consequences of information technologies in health care. An interactive sociotechnical analysis. J Am Med Inform Assoc 2007;14(5):542-9.

20. Lluch M. Healthcare professionals' organisational barriers to health information technologies. A literature review. Int J Med Inform 2001;80(12): 849-62.

21. European Union's Seventh Framework Programme for Research (FP7), ICT Challenge 5: ICT for Health, Ageing Well, Inclusion and Governance, http://cordis.europa.eu/fp7/ict/programme/challenge5_en.html

22. Hutchins E. How a cockpit remembers its speeds. Cogn Sci 1995;19(3):265-88.

23. Hollan J, Hutchins E, Kirsh D. Distributed cognition: toward a new foundation for human-computer interaction research. ACM Trans Comput Hum Interact 2000;7(2):174-96.

24. Zhang J, Patel VL. Distributed cognition, representation, and affordance. Pragmatics\& Cognition 2006;14(2):333-41.

25. Norman DA. Cognitive artifacts. Department of Cognitive Science, University of California, San Diego; 1990.

26. Kirsh D. The intelligent use of space. Artif Intell 1995;73(1):31-68.

27. Lave J. Cognition in practice: Mind, mathematics and culture in everyday life. Cambridge UniversityPress; 1988.

28. Gibson JJ. The ecological approach to visual perception. PsychologyPress; 1986.

29. Varela FJ, Thompson ET, Rosch E. The embodied mind: Cognitive science and human experience. MIT press; 1991.

30. Lakoff G, Johnson M. Philosophy in the flesh: The embodied mind and its challenge to western thought. Basic Books (AZ); 1999.

31. Clark A, Chalmers D. The extended mind. analysis 1998;58(1):7-19.

32. Schraagen JM, Verhoeven F. Methods for studying medical device technology and practitioner cognition: The case of user-interface issues with infusion pumps. J Biomed Inform 2013;46(1):181-95.

33. Norman DA. Things that make us smart: Defending human attributes in the age of the machine. Basic Books (AZ); 1993.

34. Syed S, Paul JE, Hueftlein M, Kampf M, McLean RF. Morphine overdose from error propagation on an acute pain service. Can J Anesth 2006:53(6):586-90.

35. Tariq A, Georgiou A, Westbrook J. Medication errors in residential aged care facilities: A distributed cognition analysis of the information exchange process. Int J Med Inform 2013 May;82(5):299312.

36. Pelayo S, Anceaux F, Rogalski J, Elkin P, Beuscart-Zephir MC. A comparison of the impact of CPOE implementation and organizational determinants on doctor-nurse communications and cooperation. Int J Med Inform 2012 Sep.

37. Djulbegovic B, Hozo I, Beckstead J, Tsalatsanis A, Pauker S. Dual processing model of medical decision-making. BMC Med Inform Decision Mak 2012;12(1):94.

38. Mukherjee K. A dual system model of preferences under risk. Psychol Rev 2010;117(1):243-55.

39. Kahneman D. Maps of bounded rationality: Psychology for behavioral economics. Am Econ Rev 2003;93(5):1449-75.

40. Li SY, Magrabi F, Coiera E. (2012). A systematic review of the psychological literature on interruption and its patient safety implications. J Am Med Inform Assoc 2012;19(1):6-12.

41. Park SY, Lee SY, Chen Y. The effects of EMR deployment on doctors' work practices: A qualitative study in the emergency department of a teaching hospital. Int J Med Inform 2012;81(3):204-17.

42. Zhou X, Ackerman MS, Zheng K. I just do not know why it is gone: maintaining informal information use in patient care. Proceedings of $\mathrm{CHI}$ 2009; 2009. p. 2061-70.

43. Goody J. The domestication of the savage mind. Cambridge [Eng.]; New York: Cambridge University Press; 1977.

44. Schuemie MJ, Talmon J, Moorman PW, Kors JA. Mapping the domain of medical informatics. Methods Inf Med 2009;48(1):76.

\section{Correspondence to:}

Gunnar Declerck / Xavier Aimé

INSERM, UMRS 872 équipe 20

Centre de recherche des Cordeliers, Escalier D, 2ème étage

15 , rue de l'école de médecine

75006 Paris, France

E-mail: gunnar.declerck@inserm.fr

xavier.aime@inserm.fr

\section{Appendix: Content Summary of Selected Best Papers for the IMIA Yearbook 2013, Section Human Factors and Organizational Issues ${ }^{1}$}

\section{Schraagen JM, Verhoeven F \\ Methods for studying medical device tech- nology and practitioner cognition: The case of user-interface issues with infusion pumps}

\section{J Biomed Inform 2013 Feb;46(1):181-95}

The aim of the authors was to identify the different research methods used to study user-interface issues with infusion pumps and to promote the so-called Cognitive Systems Engineering framework in order to analyze the practitioner cognition and the way it is

\footnotetext{
The complete papers can be accessed in the Yearbook's full electronic version, provided that the article is freely accessible or that your institution has access to the respective journal.
}

modified by tools. A systematic literature review was conducted (on Scopus database, from 1990 to 2011) to find papers dealing with user interface issues (or ergonomics, usability, human factors, human-machine interactions, etc.) related to infusion pumps (or intravenous pump, infusion device, patient-controlled analgesia, etc.). A total of 47 papers were selected after several exclusion steps. The papers were categorized in 6 categories (experimental comparison, case studies, etc.). An Impact Flow Diagram showing how the representations provided by the infusion pump interface impact the cognitive and collaborative behavior of practitioners was used to analyze the different methods and user-interface issues (especially failures) addressed in the papers. Finally, a case study was discussed to illustrate how the use of the diagram can support a distributed cognition analysis of infusion pump use failures. The authors conclude that most existing methods to study infusion pumps user interface issues are focused on observable behavior rather than on how artifacts shape cognition and collaboration. The generally unreflective use of computer technology by designers leads to the creation of devices with flaws in human-computer cooperation, "such as proliferating modes, making the system opaque, and providing poor feedback". These flaws "create new cognitive demands, such as increased memory demands, impaired mental models, and poor situation assessment".

\section{Tariq A, Georgiou A, Westbrook J \\ Medication errors in residential aged care facilities: A distributed cognition analysis of the information exchange process}

\section{Int J Med Inform 2013 May;82(5):299-312}

The aim of this qualitative study is to analyze the information exchange processes underlying medication ordering and drug delivery in residential aged care facilities (RACFs) in order to highlight how the dysfunctions of these processes can be the cause of medication errors. The study focuses on three Australian RACFs providing nursing and personal care full time using a mixed paper and Information and Communication Technology (ICT) system. Data was collected using minimally intrusive direct 
observations, semi-structured interviews, and artefact analysis over a period of five months. A distributed cognition framework is used to analyze information exchanges and to characterize the collective thinking that underlies the process of medication ordering and delivery. The analysis reveals four interrelated forms of cognitive processes distribution: (i) Social distribution between doctors, RACF staff and community pharmacy staff; (ii) structural distribution based on the use of cognitive artifacts (e.g. medication charts) as a mechanism to coordinate work; (iii) spatial distribution integrating different communication channels to exchange information within the organization (e.g. fax exchange of charts); (iv) temporal distribution, i.e. sequence, allocation, and synchronization of activities across time. The authors demonstrate that the medication chart "is the key cognitive artefact for maintaining inter-organisational awareness and guiding future actions in the medication process", and that its bad design (e.g. missing information elements, limited ability to present information clearly, or complication of the information exchange process due to multiple options to perform the same task), together with limitations in mutual understanding of work practices and a lack of established inter-organisational coordinating mechanisms, are the main source of coordination failures leading to medication errors. Accordingly recommendations for the design of electronic medication charts are proposed.

\section{Pelayo S, Anceaux F, Rogalski J, Elkin P, Beuscart-Zephir MC}

A comparison of the impact of CPOE implementation and organizational determinants on doctor-nurse communications and cooperation

\section{Int J Med Inform 2012 Sep 20}

This study promotes the hypothesis that organizational factors have a greater impact on doctor-nurse communications than HIT. To evaluate this claim, the authors have conducted observations $(i)$ to identify the organizational determinants of doctor-nurse communications within the medication ordering and administration process; (ii) to assess the impact of those organizational determinants and compare that impact with the impact of a Computerized Physician Order Entry (CPOE) system on doctor-nurse communications. In a first study, 60 observations (3-8 h) and 49 interviews were performed to identify the typical workplace organizations of the two organizational determinants of greatest importance to the doctor-nurse cooperation: The face-to-face communications and the co-presence of partners during action. Three organization types were identified: "common round" (face-to-face communications and presence during the partner's actions), "briefing" (face-to-face communications and no presence during the partner's actions) and "opportunistic exchanges" (no face-to-face communications and no presence during the partner's actions). In a second study, the three identified organizations were crossed with the type of record (CPOE vs. paper-based) to test their respective impact on doctor-nurse communications. Eight systematic observations in 5 different departments were performed, covering the entire medication ordering-administration process. All the doctor-nurse medication-related communications were audio-taped. The analysis of the data confirmed (i) the impact of the organizational determinants on the cooperative activities, and demonstrated (ii) that the CPOE system has no significant impact on duration, content, and quality of communications within a given workplace organization. This result is important for future studies aiming to evaluate "the (potentially negative) impact of HIT implementation on the collective and cooperative aspects of healthcare workflow", because it means they "should control for these organizational types, which serve as often hidden intermediate variables".

\section{Djulbegovic B, Hozo I, Beckstead J, Tsalatsanis A, Pauker SG \\ Dual processing model of medical decision-making}

\section{BMC Med Inform Decis Mak 2012 Sep} $3 ; 12: 94$

This paper proposes a new mathematical model of medical reasoning and decision-making based on the dual processing theory of human cognition. The model, called DSM-M (for Dual System Model applied to Medicine), postulates that medical decision is a weighted combination of the valuations from two information-processing systems: "System I", which is intuitive, automatic, fast, narrative, experiential, and affect-based; "System II", which is rational, analytical, slow, verbal, deliberative, and logical. The model is unique because it takes into account the role played by emotions in medical decision making. Especially, it postulates "that among the emotions that can influence valuation of outcomes in system I processing, regret plays an important role". The authors claim that this dual model can explain treatment decision-making when the diagnosis is partly indeterminate i.e. when a doctor has to choose treatment vs. no treatment for disease which is present with the probability $\mathrm{p}$, including phenomena as overtreatment or undertreatment which are documented in current medical practice. Two medical decision situations (treatment of pulmonary embolism and treatment of acute leukemia) are analyzed to test its applicability. The authors demonstrate that the DSM-M model can explain deviations from outcomes predicted by the standard expected utility theory (EUT) model, and account for the observed variation in management of diseases.

\section{Li SY, Magrabi F, Coiera E}

\section{A systematic review of the psychological literature on interruption and its patient safety implications}

\section{J Am Med Inform Assoc 2012 Jan- Feb;19(1):6-12}

This study proposes a systematic review of experimental studies in psychology and human-computer interaction to understand the complex effects of interruption in healthcare and identify situations when interruptions might pose a substantial risk to patient safety. The authors identify three cognitive psychology frameworks useful to analyze interruption effects (defined as occurring when a second activity requiring one's attention stops interaction with a primary task): The activation-based goal memory 
model (AGM), the prospective memory model (PM), and the multiple resource theory (MRT). A total of 63 experimental studies were retrieved using a systematic review of the literature (from 1989 to 2009) and were analyzed. Three generic task types (procedural, problem-solving, and decision-making) were identified together with 12 independent variables influencing the effects of interruption on patient safety and task efficiency. Of the 12 variables, 6 were identified as the most important based on their centrality to the network of interruption variables and the number of studies: Working memory load, interruption similarity, interruption position, interruption modality, practice/experience, and interruption-handling strategies. A negative effect of interruption on task completion time, resumption lag, work strategy, decision-making process, and error, was documented by the studies analyzed. Based on these findings, several recommendations to minimize the disruptive effects of interruption in clinical settings are proposed: e.g., to avoid interruptions when engaged in task positions of high working memory demands, train clinical staff in interruption-handling strategies, or provide environmental cues to aid recovery from interruptions.

\section{Park SY, Lee SY, Chen Y}

\section{The effects of EMR deployment on doctors' work practices: a qualitative study in the emergency department of a teaching hospital Int J Med Inform 2012 Mar;81(3):204-17}

The aim of this qualitative study was to analyze how the design of electronic medical records (EMR) systems affects medical work practices by observing the rollout of the EMR in situ during the paper-to-electronic transition period. The study focuses on the rollout of electronic medical (MD) notes and documentation practices in an emergency department (ED). The pre-, during, and post-EMR deployment periods were studied using qualitative field study methods. A total of $106 \mathrm{~h}$ of field observations and 8 semi-structured interviews were conducted over a period of 6 months. The analysis of data shows that the electronic MD notes system shifted documentation workload, caused workflow changes among ED doctors, and influenced the way ED doctors interacted with nurses and patients: (i) Time spent for documentation task increased four to five fold, which in turn significantly increased the number of incomplete charts; (ii) The use of the EMR directly affected the division of labor between attending and residents, shifting more documentation responsibilities to residents; (iii) The deployment of the electronic MD notes also changed the location of documentation and led to use of paper notes as documentation aids to transfer information from the patient bedside to the charting room; (iv) It increased the cognitive load of doctors, aggravated multi-tasking due to flexibility in the system use allowing more interruptions; (v) Finally, it caused ED doctors' work to become largely stationary in the charting room, which further contributed to reducing the time spent with patients and the interaction with nurses. Based on those observations, several recommendations are made for future EMR systems design: (1) To provide functionalities supporting note-intensive tasks, i.e. time-consuming tasks requiring detailed documentation (e.g. physical exams, medical treatments, entering orders, admitting and discharging patients); (2) to define different roles explicitly in the collaborative documentation process between residents and attending to avoid unfair work redistribution; (3) to balance flexibility and interruptions in documentation work, to avoid increased multi-tasking and simultaneous management of more unfinished patient charts. 